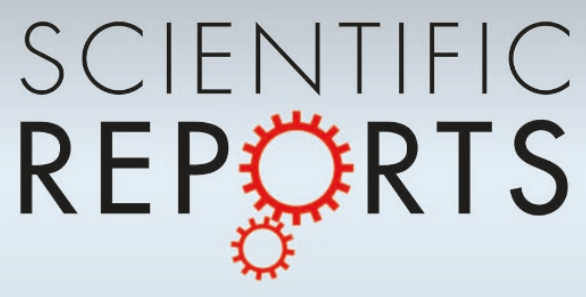

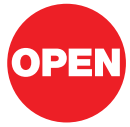

SUBJECT AREAS:

METHODS OF

TOXICOLOGY STUDIES

CELL-PARTICLE INTERACTIONS

MEDICAL TOXICOLOGY

IMAGING TECHNIQUES AND

AGENTS

Received

18 November 2012

Accepted

10 December 2012

Published

24 January 2013

Correspondence and requests for materials should be addressed to

A.P.-M. (prinamea@

tcd.ie)

* These authors contributed equally to this work.

\section{Citrullination as early-stage indicator of cell response to Single-Walled Carbon Nanotubes}

\author{
Bashir Mustafa Mohamed ${ }^{*}$, Dania Movia ${ }^{1,2 *}$, Anton Knyazev³, Dominique Langevin ${ }^{3}$, \\ Anthony Mitchell Davies' ${ }^{1}$ Adriele Prina-Mello ${ }^{1,2 *} \&$ Yuri Volkov ${ }^{1,2}$
}

'Department of Clinical Medicine, Trinity College Dublin, Ireland, ${ }^{2}$ Centre for Research on Adaptive Nanostructures and
Nanodevices, Trinity College Dublin, Ireland, ${ }^{3}$ Universite Paris 11 -CNRS, Laboratoire de Physique des Solides, France.

Single-walled carbon nanotubes (SWCNTs) have been widely explored as potential technologies for information systems and medical applications. The impact of SWCNTs on human health is of prime concern, if SWCNTs have a future in the manufacturing industry. This study proposes a novel, inflammation-independent paradigm of toxicity for SWCNTs, identifying the protein citrullination process as early-stage indicator of inflammatory responses of macrophages (THP-1) and of subtle phenotypic damages of lung epithelial (A549) cells following exposure to chemically-treated SWCNTs. Our results showed that, while most of the cellular responses of A549 cells exposed to SWCNTs are different to those of similarly treated THP-1 cells, the protein citrullination process is triggered in a dose- and time-dependent manner in both cell lines, with thresholds comparable between inflammatory (THP-1) and non-inflammatory (A549) cell types. The cellular mechanism proposed herein could have a high impact in predicting the current risk associated with environmental exposure to SWCNTs.

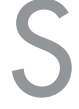

ingle-walled carbon nanotubes (SWCNTs) represent a relatively newly discovered allotrope of carbon ${ }^{1}$ having a wide range of potential applications in the information and communications technology (ICT) $)^{2,3}$ and medicine ${ }^{4-7}$ fields.

As for many other nanomaterials, several aspects of the effects of SWCNTs to human health are poorly defined $^{8}$. From 2006 onwards, the number of reports investigating in vitro $0^{9,10}$ and in vivo ${ }^{11-13}$ the lung toxicity of SWCNTs has increased exponentially but the current knowledge of SWCNTs' adverse effects in the lungs is still fragmentary, and sometimes contradictory ${ }^{8,14,15}$. This fragmentation originates from three main aspects ${ }^{16}$. Firstly, the studies exploring SWCNTs' toxicity are not standardised for SWCNTs produced by different methods and do not employ equivalent testing models. Secondly, in many cases such experiments do not accurately reflect actual exposure conditions. This originates ultimately from the absence of environmental exposure data on SWCNTs and of specific information regarding the end-of-life/disposal of SWCNTs-based devices from ICT and medical applications. Thirdly, specific cellular mechanisms through which SWCNTs may exert their adverse toxic effects in vivo are still under debate.

By looking at the emerging picture, the most widely accepted paradigms ${ }^{14}$ for SWCNTs' toxicity are (a) the oxidative stress ${ }^{13,17,18}$ (due to redox features of this nanomaterial ${ }^{19}$ or of transition metals and other environmental contaminants present in SWCNTs ${ }^{18,20}$ ) and (b) inflammation (associated with inflammatory and fibrogenic responses in the lungs ${ }^{21}$ and ineffective recognition of SWCNTs by macrophages ${ }^{22}$. This study proposes a potential novel paradigm according to which SWCNTs triggered a toxic response by activating an inflammation-independent cellular mechanism. Citrullination (i.e., the enzymatic conversion of protein-contained arginine to citrulline carried out by peptidylarginine deiminase (PAD)) was identified as an early-stage indicator of such inflammation-independent mechanism. Macrophages (THP-1 cells) and lung epithelial (A549) cells were exposed to chemically-treated SWCNTs and the protein citrullination levels were quantified. Cellular protein modification levels were then correlated to cytotoxic cellular responses and to the secretion of pro-inflammatory cytokines.

Citrullination is known as an inflammation associated process ${ }^{23}$ characteristic of extra-articular manifestations of rheumatoid arthritis (RA) ${ }^{24-26}$, such as interstitial pneumonia and rheumatoid nodules. Our group has recently demonstrated that exposure to various nanomaterials does induce citrullination ${ }^{27}$, which might ultimately trigger autoimmune diseases. Protein citrullination, as well as $\mathrm{Ca}^{2+}$-mediated PAD activation, was in fact detected in 
cultured human cells (A549 and THP-1 cells) and in mouse lung tissue after exposure to nanosized amorphous silicon dioxide $\left(\mathrm{SiO}_{2}\right)$, ultra-fine carbon black (ufCB) and SWCNTs ${ }^{27}$.

Here, we present for the first time evidence that SWCNTs are able to induce protein citrullination in a lung epithelial cell line prior to any detectable onset of inflammatory responses. SWCNTs were tested following chemical treatment, since chemical modifications are known to reduce the amount of toxic metal impurities (used in the production of the carbon nanomaterials and present in the asproduced, pristine SWCNTs) that can ultimately induce oxidative stress and cytokine secretion in cells exposed to SWCNTs. Furthermore, chemical modifications are fundamental to increasing the manufacturability and biocompatibility of SWCNTs for ICT and medical applications, thus making our testing model relevant when compared to realistic exposure scenarios of the manufacturing industry. Our results clearly indicated that exposure to SWCNTs induced citrullination in THP-1 and A549 cells after 6 h. In particular, high citrullination levels were detected in lung epithelial cells, which are not responsible for inflammation responses, thus ultimately advocating citrullination as an inflammation-independent process and highlighting the potential use of citrullination as earlystage indicator of an emerging inflammation-independent paradigm of SWCNTs' toxicity.

\section{Results}

High content screening and analysis (HCSA). Protein citrullination. Human lung epithelial (A549) cells and phagocytic (THP-1) cells were exposed to various SWCNTs concentrations (1, $5,10 \mu \mathrm{g} / \mathrm{ml}$ ) for $6 \mathrm{~h}$ and $24 \mathrm{~h}$, and HCSA was carried out to quantify the induction of protein citrullination. The data (Figure 1A) indicated a significant increase in protein citrullination in both cell lines in a time- and dose-dependent manner, with maximum effects seen at $24 \mathrm{~h}$ and at the highest dose $(10 \mu \mathrm{g} / \mathrm{ml})$ tested. Immunofluorescent images of cells exposed to chemically-treated SWCNTs (Figure 1B) showed citrullinated proteins in the cytoplasm and around the nucleus after 24 exposure, thus validating that citrullinated proteins were expressed.

By increasing the complexity of the SWCNT's functionalization ( -SWCNTs $<$ p SWCNTs/BSA $<$ f-SWCNTs $<$ f-SWCNTs/BSA), the induction of citrullinated proteins decreased. High citrullination levels were found in fact when cells were treated with p-SWCNTs, while cells exposed to f-SWCNTs/BSA showed low citrullination levels. However, high levels of citrullination were detected after exposure to Mal-SWCNTs/BSA. This could be associated with the presence of cytotoxic maleic moieties on the nanotubes surface.

Interestingly, significantly high citrullination was observed in A549 cells (which are cells not directly involved in inflammation responses) treated with SWCNTs. In detail, citrullination was detectable in SWCNTs-treated A549 cells even after $6 \mathrm{~h}$ exposure (Figure 1A). This suggested that the induction of protein citrullination could be considered as a sign of early cellular damage in an in vitro, non-inflammatory model such as A549 cells.

Cytotoxicity responses. Three different concentrations $(1,5,10 \mu \mathrm{g} /$ $\mathrm{ml}$ ) of SWCNTs were tested on THP-1 and A549 cell populations at three time points $(3,6,24 \mathrm{~h})$. Cell count reduction, cell membrane permeability, lysosomal mass/pH and nuclear morphology changes were the cell parameters monitored by HCSA (Figure 2).

Cell count reduction. A dose- and time- dependent reduction of cell viability was detected when THP-1 cells were exposed to SWCNTs (Figure 2). In detail, significant reduction in cell count was seen after $3 \mathrm{~h}$ exposure to $\mathrm{p}$-SWCNTS/BSA and f-SWCNTS/BSA, and after $6 \mathrm{~h}$ exposure to p-SWCNTs, f-SWCNTs and Mal-SWCNTs/BSA. In contrast, A549 cells showed a decreased cell count only when exposed to the highest concentration $(10 \mu \mathrm{g} / \mathrm{ml})$ of $\mathrm{p}$-SWCNTs and f-SWCNTs for $24 \mathrm{~h}$.

Cell membrane permeability. It has been shown that alterations of the cellular membrane permeability are often associated with an ongoing toxic or apoptotic cell responses (21). A clear dose-dependent
A

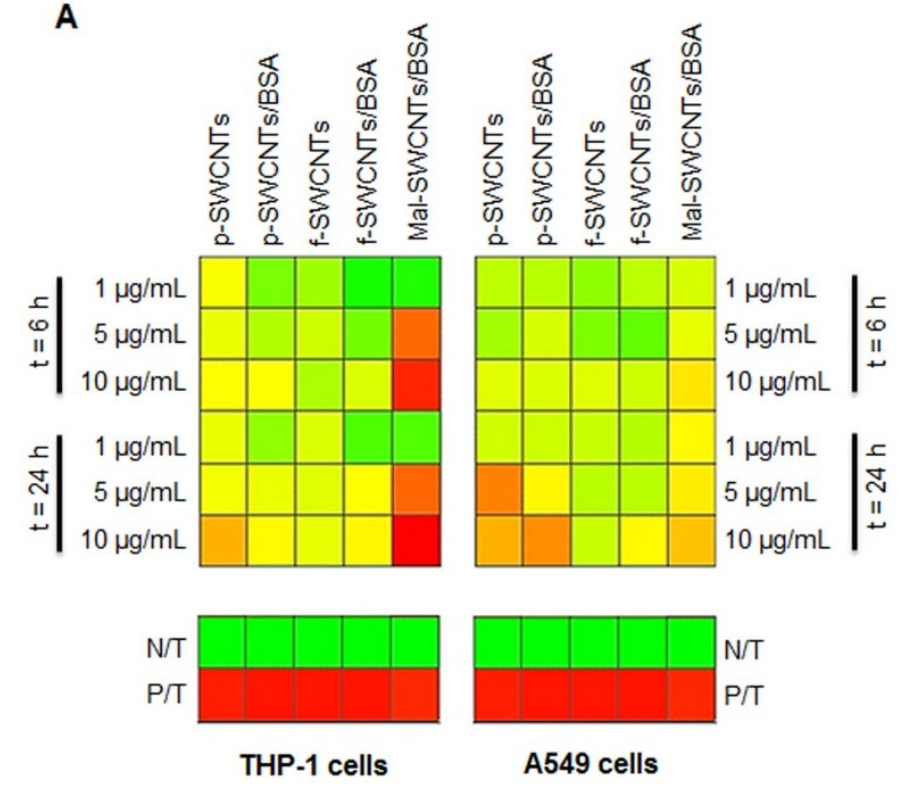

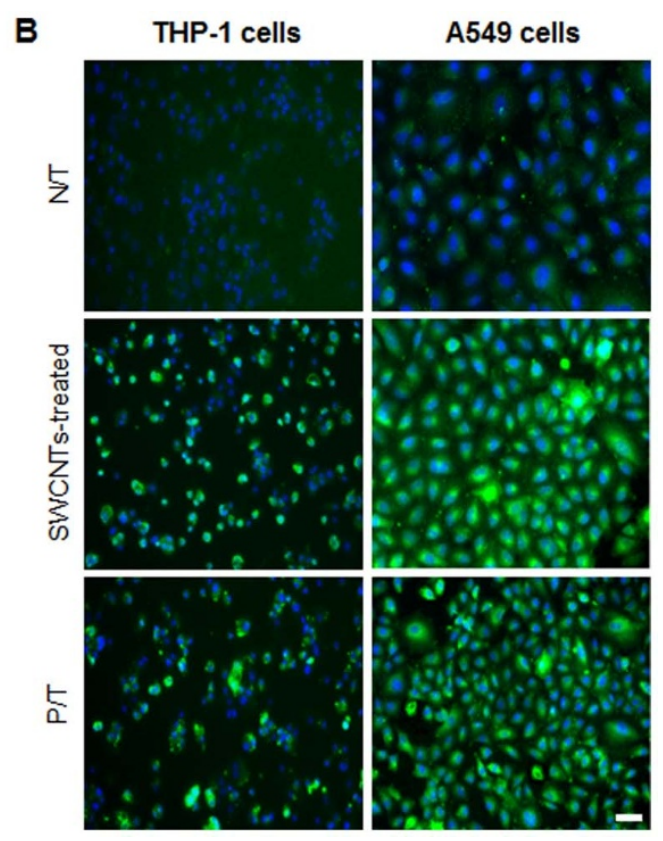

Figure $1 \mid$ Citrullination levels in THP-1 and A549 cells exposed to chemically-treated SWCNTs (p-SWCNTs, p-SWCNTs/BSA, f-SWCNTs, fSWCNTs/BSA and Mal-SWCNTs/BSA) for 6 and $24 \mathrm{~h}$. (A) Graphical tables (heatmaps) reflect the citrullination levels ranging from dark green (lower than $15 \%$ change from the maximum value measured) to bright green (30\%), yellow (50\%), bright orange (60\%), dark orange (75\%) and finally red (higher than $75 \%$ change from the maximum value). (B) Representative fluorescent images of untreated (N/T), SWCNTs-treated and PAD-treated (P/T) THP-1 and A549 cells after 24 h exposure. Cells were immunostained for citrulline expression (in green) and nuclei (in blue). SWCNTs sample: pSWCNTs; scale bar: $40 \mu \mathrm{m}$ (10× magnification). 


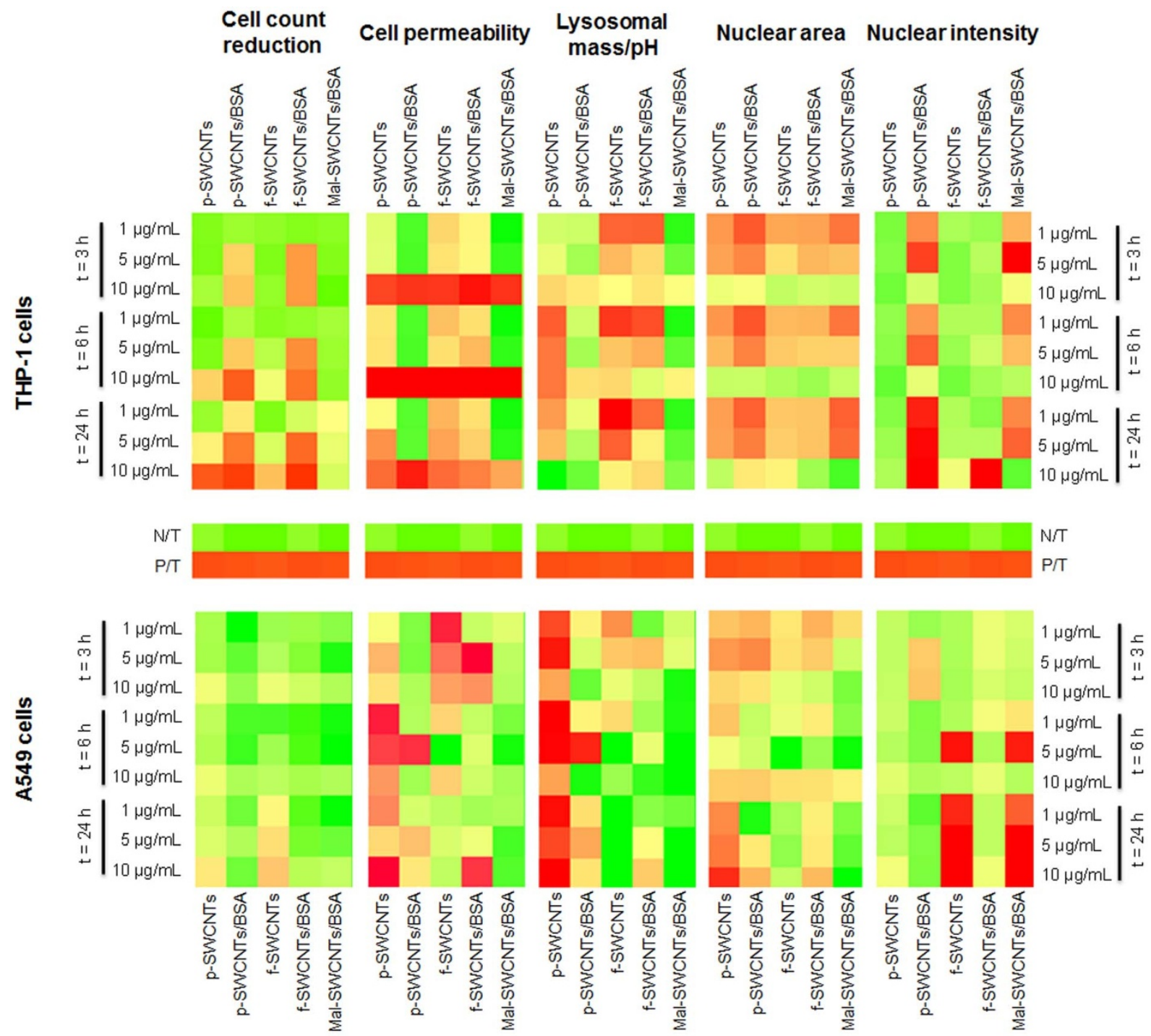

Figure $2 \mid$ Graphical tables (heatmaps) of the cytotoxicity data of THP-1 and A549 cells exposed to p-SWCNTs, p-SWCNTs/BSA, f-SWCNTs, fSWCNTs/BSA and Mal-SWCNTs/BSA for 3, 6 and $24 \mathrm{~h}$. Colorimetric gradient tables reflect the changes in cell count reduction, cell membrane permeability, lysosomal mass $/ \mathrm{pH}$, nuclear area and nuclear intensity. Colours range from dark green (values lower than $15 \%$ change from the maximum value measured) to bright green (30\%), yellow (50\%), bright orange (60\%), dark orange (75\%) and finally to red (values higher than $75 \%$ change from maximum value). Heatmap values are normalised to the percentages of the positive control (P/C) and Z-score is calculated as described in the statistical analysis section. Data represent two independent experiments performed in triplicate samples.

increase in cell membrane permeability was observed in both THP-1 and A549 cells when exposed to p-SWCNTs, f-SWCNTs and fSWCNTs/BSA (Figure 2), whereas p-SWCNTs/BSA and MalSWCNTs/BSA caused significant cell membrane permeability changes only at the highest concentration $(10 \mu \mathrm{g} / \mathrm{ml})$ in both cell lines.

Lysosomal mass/pH. Lysosomal mass/pH changes were significant when THP-1 cells were exposed to p-SWCNTs, f-SWCNTs and fSWCNTs/BSA, while subtle lysosomal mass/pH changes were detected in THP-1 cells exposed to p-SWCNTs/BSA and Mal-SWCNTs/ BSA (Figure 2). Lysosomal mass/pH was also markedly altered in A549 cells, with the lowest response associated with the exposure to Mal-SWCNTs/BSA.

Nuclear area and nuclear intensity. Since changes in the nuclear morphology can be an additional sign of cell stress and apoptotic/ toxic stimuli, the nuclear size and intensity of THP-1 and A549 cells were monitored after exposure to SWCNTs for extended exposure intervals $(24 \mathrm{~h})$. We observed significant nuclear morphological changes in SWCNTs-treated THP-1 cells, while the nuclear morphology was only marginally altered in A549 cells exposed to SWCNTs (Figure 2).

Cytokines secretion. To further explore the possible interdependence between the citrullination process and the inflammatory changes in cells of different origin as triggered by exposure to chemicallytreated SWCNTs, the secretion of the pro-inflammatory cytokines Tumour Necrosis Factor-alpha (TNF- $\alpha$ ) and Interleukin-6 (IL-6) was quantified after treatment of inflammatory (THP-1 cells) and non-inflammatory (A549) cells with such nanomaterials.

TNF- $\alpha$ and IL- 6 are known to be among the most important cellsignalling protein molecules secreted by activated macrophages (such as PMA-activated THP-1 cells) during inflammation ${ }^{28}$. Figure 3 and Figure 4 display the amount of TNF- $\alpha$ and IL- 6 secreted by THP-1 and A549 cells exposed for 6 and $24 \mathrm{~h}$ to increasing concentrations $(1,5$ and $10 \mu \mathrm{g} / \mathrm{ml})$ of p-SWCNTs, p-SWCNTs/BSA, fSWCNTs, f-SWCNTs/BSA, Mal-SWCNTs/BSA. For THP-1 cells, we observed that the secretion resulted to be time- and dosedependent. Interestingly, a higher secretion of TNF- $\alpha$ and IL- 6 was 

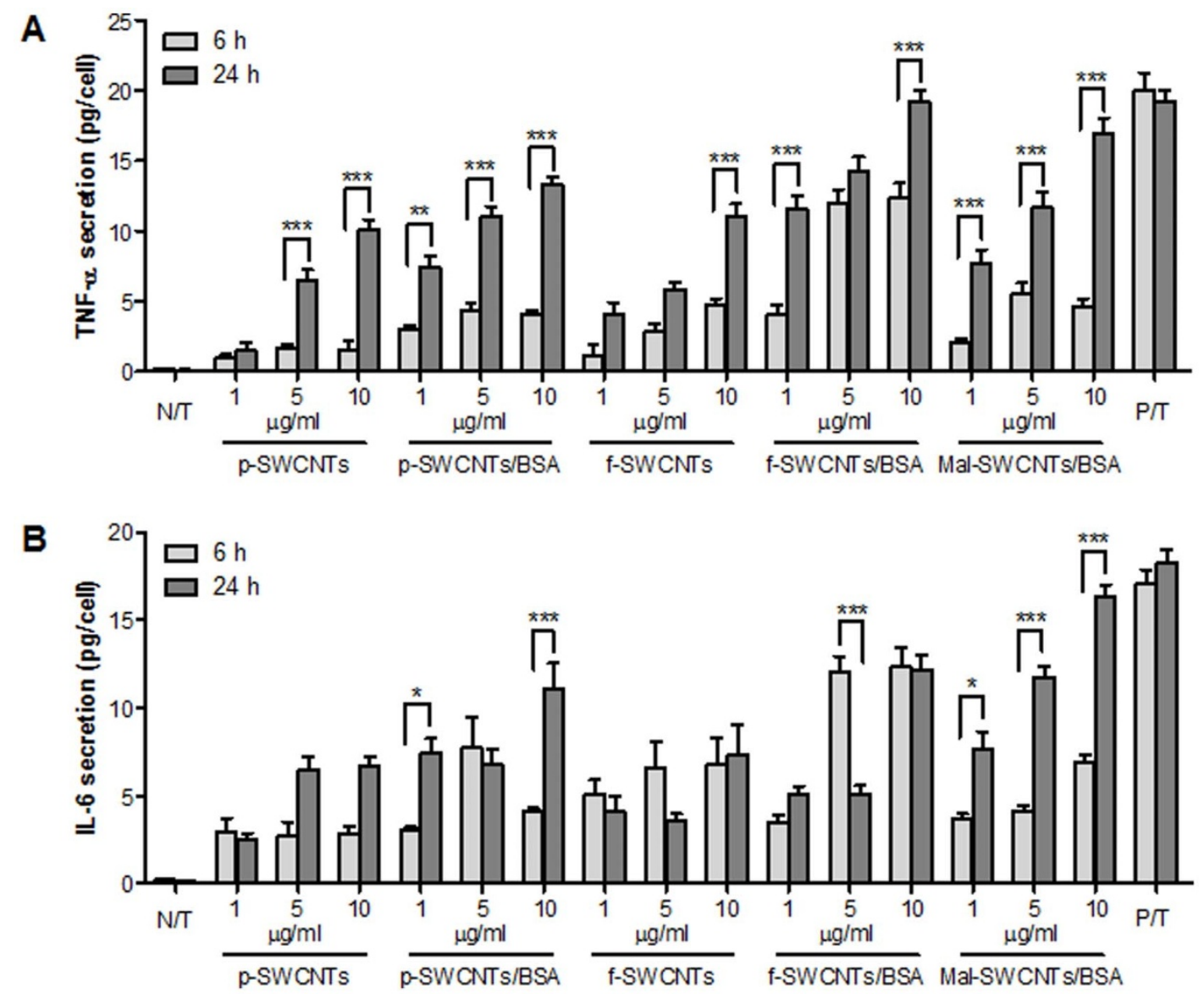

Figure 3 Release of (A) TNF- $\alpha$ and (B) IL-6 from THP-1 cells after 6 h (light grey) and $24 \mathrm{~h}$ (dark grey) exposure to p-SWCNTs, p-SWCNTs/BSA, fSWCNTs, $\mathrm{f}-\mathrm{SWCNTs} / \mathrm{BSA}$ and Mal-SWCNTs/BSA at various concentrations $(\mathbf{1}, \mathbf{5}$ and $10 \mu \mathrm{g} / \mathrm{mL})$. The symbols $\left({ }^{*}\right),\left({ }^{* *}\right)$ and $\left({ }^{* * *}\right)$ indicate significant time-dependent changes $(p<0.05, p<0.01$ and $p<0.001$, respectively).

detected when SWCNTs were conjugated with BSA (p-SWCNTs/ BSA, f-SWCNTs/BSA and Mal-SWCNTs/BSA), while lower levels of TNF- $\alpha$ and IL- 6 were detected after exposure to unconjugated SWCNTs (p-SWCNTs and f-SWCNTs). This phenomenon could be associated with the higher de-bundling of BSA-conjugated SWCNTs, which might have triggered the onset of acute phase reaction.

In contrast, exposure of A549 cells to SWCNTs did not significantly trigger the secretion of TNF- $\alpha$ (Figure 4A) or IL-6 (Figure 4B) over 6 and $24 \mathrm{~h}$ exposure, leaving the levels of the secreted proteins comparable to those of the untreated cell culture $(\mathrm{N} / \mathrm{T})$.

\section{Discussion}

Citrullination has been reported to be a process present in a wide range of inflammatory tissues, suggesting that this is an inflammation-dependent rather than disease-dependent process $^{23}$. Our group has previously demonstrated that nanomaterials of different origin are capable of promoting specific transformation of the amino acid arginine into the molecule called citrulline ${ }^{27}$, which can lead ultimately to the development of autoimmune conditions such as rheumatoid arthritis (RA) ${ }^{14}$. Here, we showed for the first time that exposure to differently processed SWCNTs (in terms of the chemical treatment applied and functionalities/chemical compounds decorating their surface) significantly influenced the protein citrullination levels in cultured THP-1 cells as well as in non-inflammatory lung epithelial cells (Figure 1), thus suggesting citrullination as an earlystage marker of a novel inflammation-independent paradigm for SWCNTs toxicity.
THP-1 and A549 cells were selected as the most relevant in vitro models for our study. THP-1 cells were employed to represent the resident phagocytic cells that have the main function of removing pathogens, senescent cells and external particles from the lungs ${ }^{29,30}$, while A549 cells represented an in vitro model for lung alveoli cells, which are not associated with inflammatory responses. A549 cells have been extensively used for assessing pulmonary cytotoxicity, including nanomaterials-induced cytotoxicity ${ }^{10,31,32}$.

Our results showed that, following exposure to SWCNTs, high levels of citrullination were found in cultured A549 cells, as well as in THP-1 cells, independently of the chemical treatment applied to SWCNTs (Figure 1). Furthermore, we found that, even if protein citrullination occurred, the cytotoxic (Figure 2) and inflammatory (Figure 4) cellular responses were significantly lower in SWCNTstreated A549 cells than in similarly treated THP-1 cells (Figure 2 and Figure 3, respectively). Similarly to a previous study published by $\mathrm{Hu}$ et al. ${ }^{33}$, showing that lymphocytes were more sensitive to SWCNTs as compared to human lung cells, our data proved that phagocytic THP-1 cells were highly sensitive to all SWCNTs samples (Figure 2), responding to the SWCNTs exposure with high levels of secreted pro-inflammatory cytokines (TNF- $\alpha$ and IL-6) (Figure $3 \mathrm{~A}$ and Figure 3B, respectively). In contrast, A549 cells were only marginally affected by the exposure to the chemically-treated SWCNTs (Figure 2). Additional analysis of the inflammatory mediators did not show in fact any significance secretion of TNF- $\alpha$ and IL- 6 from A549 cells exposed to SWCNTs (Figure 4). These results, together with literature data showing that SWCNTs can lead to the suppression of a variety of inflammatory mediators (including IL-6) in in vitro lung epithelial cell models ${ }^{34}$, provide evidence that SWCNTs 
A
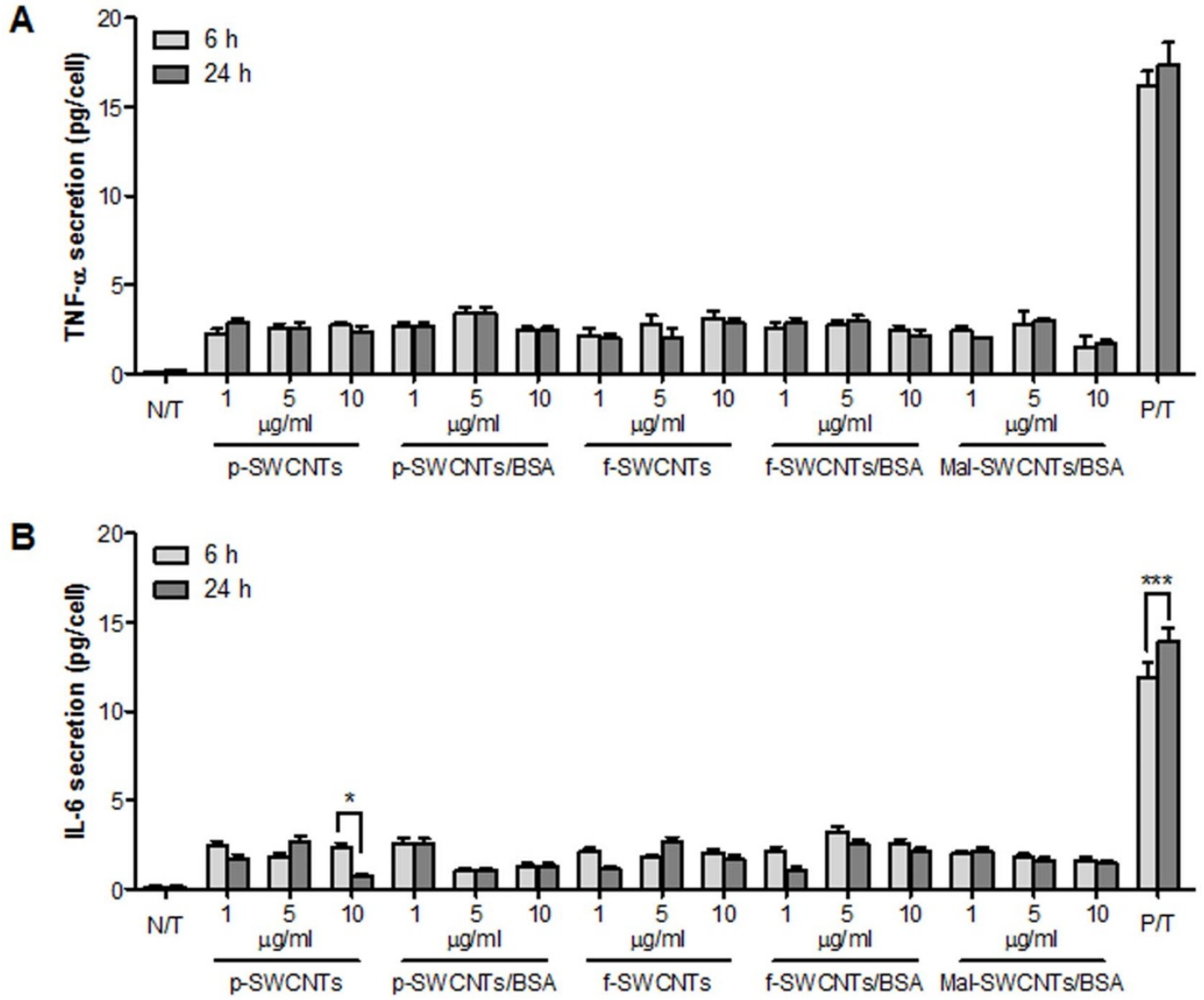

Figure $4 \mid$ Release of (A) TNF- $\alpha$ and (B) IL-6 from A549 cells after $6 \mathrm{~h}$ (light grey) and $24 \mathrm{~h}$ (dark grey) exposure to p-SWCNTs, p-SWCNTs/BSA, f-SWCNTs, f-SWCNTs/BSA and Mal-SWCNTs/BSA at various concentrations $(1,5$ and $10 \mu \mathrm{g} / \mathrm{mL})$. The symbols $\left({ }^{*}\right)$ and $(* * *)$ indicate significant time-dependent changes ( $p<0.05$ and $p<0.001$, respectively).

could be possibly cytotoxic to lung epithelial cells by activating an inflammation-independent cellular mechanism. Keeping in mind that citrullination of proteins is a process distinct from the formation of the free amino acid citrulline as by-product of oxidative stress enzymes, our results suggest citrullination as a potential early-stage marker for a novel paradigm of SWCNTs' toxicity.

In conclusion, our study showed for the first time that increased increased protein citrullination can be a potential robust indicator

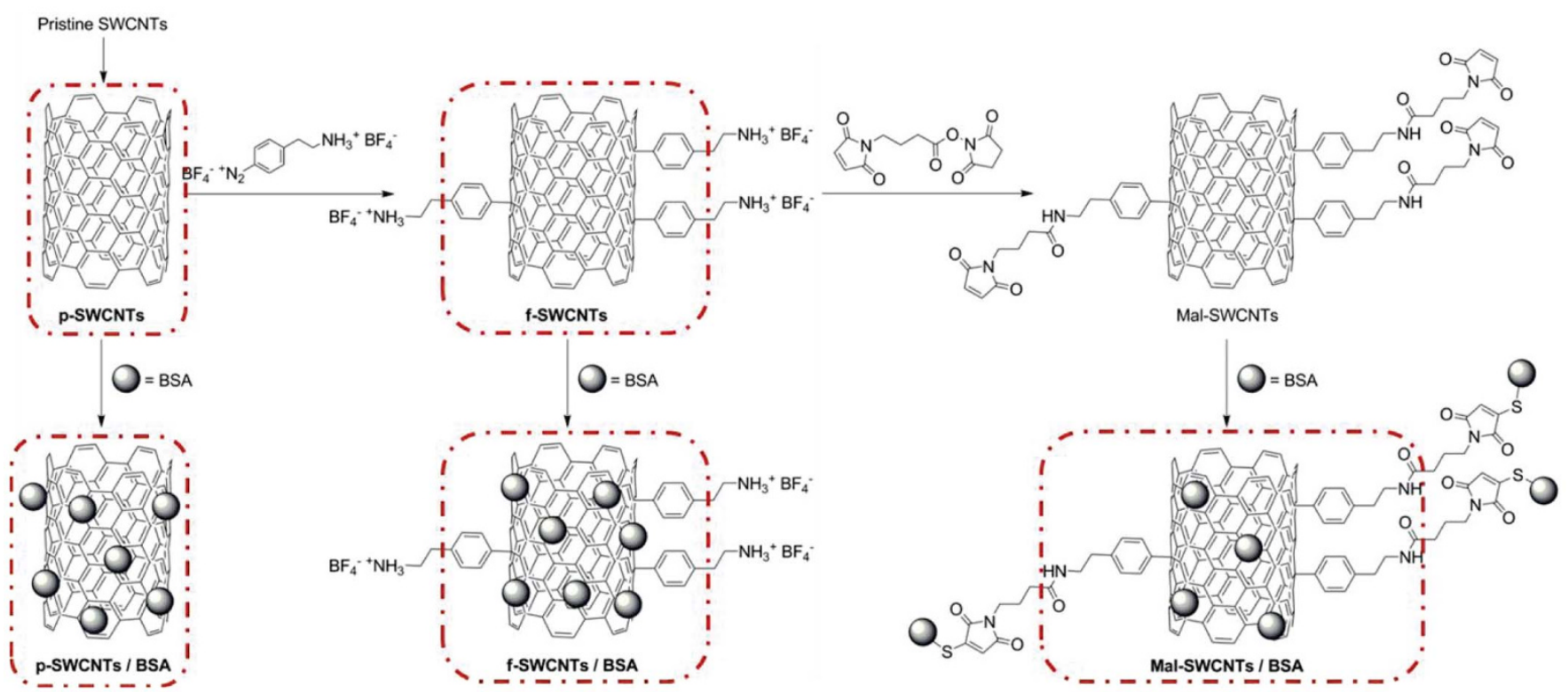

Figure 5 Schematic of the purification and covalent functionalization of pristine SWCNTs. Boxes highlight the SWCNTs samples tested in this study. Figure adapted from Knyazev et al..$^{35}$. 
for a novel inflammation-independent paradigm defining the SWCNTs lung toxicity. Given that exposure to SWCNTs triggered an inflammatory response of THP-1 cells, including markedly increased levels of secreted pro-inflammatory cytokines, one could assume that citrullination occurred as a down-stream process of the inflammatory response to SWCNTs. However, our results in a noninflammatory, in vitro cell model (such as A549 cells) showed that protein citrullination was not necessarily an inflammation-dependent process. In detail, small changes in cytotoxic cellular responses and low levels of cytokines secretion were associated with relatively high levels of protein citrullination in A549 cells exposed to five different types of SWCNTs.

Our results strongly advocate for a novel inflammation-independent paradigm of SWCNTs toxicity and the use of citrullination levels as an early-stage indicator for the hazard ranking of SWCNTs and potentially of nanomaterials, more in general. Further studies are required to fully explore the mechanism(s) involved in the citrullination induced by exposure to SWCNTs in vitro and in in vivo correlation studies.

\section{Methods}

Materials. Chemicals and solvents were purchased from commercial sources (SigmaAldrich, Fisher Scientific, Invitrogen and Calbiochem). Pristine SWCNTs prepared by laser ablation were purified and functionalized following the procedure reported in a previous study ${ }^{35}$ (Figure 5). Briefly, pristine SWCNTs were first purified (pSWCNTs) and then functionalized by adding 4-(2-aminoethyl)benzenediazonium tetrafluoroborate, thus obtaining covalently functionalized single-walled carbon nanotubes (f-SWCNTs). The f-SWCNTs nanotubes were then treated with 4maleimidobutyric acid N-hydroxysuccinimide ester, obtaining Mal-SWCNTs. The resulting p-SWCNTs, f-SWCNTs and Mal-SWCNTs derivatives were exposed to a solution of bovine serum albumin (BSA) in 4-(2-hydroxyethyl)-1-

piperazineethanesulfonic acid (HEPES), thus yielding p-SWCNTs/BSA, f-SWCNTs/ BSA and Mal-SWCNTs/BSA, respectively. Table S23 in the Supporting Information reports a summary of the characterization of the SWCNTs samples (average atomic force microscopy height measurements, $\mathrm{O}_{2}$ atomic percentage and zeta potential of SWCNT dispersions in water at $\mathrm{pH} 7$ ).

Cell culture. Human lung epithelial (A549) and phagocytic (THP-1) cell lines (ATCC, Manassas, VA, USA) were cultured in DMEM and RPMI 1640 medium, respectively. Both the media were supplemented with $10 \%(\mathrm{v} / \mathrm{v})$ foetal bovine serum (FBS) and 1\% (v/v) L-glutamine/penicillin/streptomycin. Cells were grown in a humidified incubator at $37^{\circ} \mathrm{C}$ in $5 \% \mathrm{CO}_{2}$.

Cell plating and exposure. For experimentation, A549 and THP- 1 cells were seeded in 96-well plates at a concentration of 5,000 and 15,000 cells/well, respectively (Nunc Inc., USA). THP- 1 cells were stimulated with $25 \mathrm{ng} / \mathrm{ml}$ of phorbol 12 -myristate 13-acetate (PMA) for $72 \mathrm{~h}$ before exposure to SWCNTs. THP-1 and A549 cells were exposed to five different samples of chemically-treated SWCNTs, namely pSWCNTs, p-SWCNTs/BSA, f-SWCNTs, f-SWCNTs/BSA and Mal-SWCNTs/BSA. Exposure concentrations ranged from $1 \mu \mathrm{g} / \mathrm{ml}$ to $10 \mu \mathrm{g} / \mathrm{ml}(1,5$ and $10 \mu \mathrm{g} / \mathrm{ml})$.

High content screening and analysis (HCSA). HCSA combines high-resolution digital imaging with powerful software algorithms to enhance the quantitative data processing. HCSA is currently recognised as the industry standard for drug screening. Recently, HCSA has also been implemented for the analysis of cell changes induced by nanomaterials ${ }^{27,36-39}$ since this is the only contemporary technique which enables to handle large experimental data sets correlating cell responses to multiple nanomaterials, reagents, concentrations and data points. HCSA was used to quantify citrullination process and cytotoxic responses following exposure to SWCNTs.

- Protein citrullination: Cells were exposed to SWCNTs for $6 \mathrm{~h}$ and $24 \mathrm{~h}$ and then fixed using $3 \%$ paraformaldehyde (PFA), as previously described ${ }^{27}$. After gentle washing with phosphate buffer solution (PBS), cells were incubated with anticitrulline antibody (Cat. No: ab6464, 1:200 dilution) for $1 \mathrm{~h}$ at room temperature. Cells were washed three times with PBS and then incubated with FITClinked goat anti-rabbit antibody for $1 \mathrm{~h}$ and stained for nuclei with Hoechst 33342. Plates were scanned using IN Cell Analyzer 1000 automated microscope (GE Healthcare, Buckinghamshire, UK). Images were acquired in a stereology configuration of five randomly selected fields per well at $10 \times$ magnification using two detection channels. Protein citrullination was quantified using the dual area object analysis module of the IN Cell Investigator software (GE Healthcare, Buckinghamshire, UK). The module allows for simultaneous quantification of subcellular inclusions that are marked by different fluorescent labels and measures fluorescence intensity associated with predefined nuclear and cytoplasmic compartments. Cells exposed to peptidylarginine diminase (PAD) were used as positive control $(\mathrm{P} / \mathrm{T})$.
- Cytotoxicity assay: Following exposure to SWCNTs for 3,6 and $24 \mathrm{~h}$, a multiparametric cytotoxicity assay was performed using HCS reagent HitKit ${ }^{\mathrm{TM}}$ as per manufacturer's instructions (Thermo Fisher Scientific Inc., USA). Briefly, this kit enable to measure cell viability, cell membrane permeability and lysosomal mass/ $\mathrm{pH}$, which are toxicity-linked cellular markers. The experimental layout for the automated microscopic analysis, based on the In Cell Analyzer 1000, was composed of untreated cells (negative control or N/T), cells treated with chemicallymodified SWCNTs and cells exposed to cisplatin (positive control or P/T), which is a platinum-based cytostatic drug used to treat various types of cancers ${ }^{40}$. Images were acquired in a stereology configuration of five randomly selected fields at $10 \times$ magnification using three detection channels with different excitation filters. The rate of cell viability and proliferation were assessed by the automated analysis of the nuclear count and morphology (DAPI filter); in parallel the fluorescent staining intensities reflecting cell permeability (FITC filter) and lysosomal mass/ $\mathrm{pH}$ changes (TRITC filter) were also quantified for each individual cell present in the examined microscopic fields (IN Cell Investigator, GE Healthcare, UK).

Cytokines secretion. The expression of pro-inflammatory cytokines (Tumour Necrosis Factor-alpha (TNF- $\alpha$ ) and Interleukin-6 (IL-6)) expressed by THP-1 and A549 cells exposed to chemically-treated SWCNTs was quantified by Enzyme-Linked Immunosorbent Assays (ELISAs) (Human TNF- $\alpha /$ TNFSF1A and Human IL-6 DuoSet ELISA kit, R\&D Systems, Minneapolis, USA), according to the manufacturer's manual. The assays were carried out in duplicates. The optical density of each well at $450 \mathrm{~nm}$ was determined by means of an Epoch microplate reader (Biotek, USA), calibrated against standards and corrected by subtracting the optical aberration of the 96 -well plastic plate at $540 \mathrm{~nm}$. Cells were counted using HCSA and Trypan Blue exclusion assay in order to quantify the cytokine production as picograms per cell (pg/cell) at the different exposure concentrations and time points. Cell exposed to LPS were used as positive control (P/T).

Statistical analysis. A two-way analysis of variance (ANOVA) followed by a Bonferroni post-test analysis was carried out for all HCSA and ELISA assays (Prism; Graph-Pad Software Inc., USA). $p<0.05$ was considered statistically significant. The entire list of $p$ values can be found as Supplementary Tables S1-S22 online. HCSA and ELISA data, as well as the protein citrullination results, are presented as mean values $\left(\mathrm{n}_{\text {test }}=2\right) \pm$ standard error of the mean and normalized to the negative control. Due to the large amount of information acquired by HCSA, a data mining and exploration platform was used (KNIME (http://KNIME.org, 2.0.3) in combination with a HiTS screening module (http://code.google.com/p/hits 0.3.0) in order to screen and normalize all parameters under investigation as previously reported ${ }^{27,36-38}$. All measured parameters were normalized using the percentages of the positive controls. $Z$-score was used for scoring the normalized values. These scores were summarized using the mean function as follows: $\mathrm{Z}$-score $=(\mathrm{x}$-mean $) / \mathrm{SD}$. Heatmaps (i.e., graphical illustration in a colorimetric gradient table format) were adopted as the most suitable schematic representation to report on any statistical significance and variation from normalized controls based on their Z-score value. Heatmap tables illustrate the range of variation of each quantified parameter from the minimum (green), through the mean (yellow), to the maximum (red) value.

1. Hirsch, A. The era of carbon allotropes. Nat Mater 9, 868-871 (2010).

2. Sekitani, T. \& Someya, T. Stretchable, large-area organic electronics. Adv Mater 22, 2228-2246 (2010).

3. Wei, L., Tezuka, N., Umeyama, T., Imahori, H. \& Chen, Y. Formation of singlewalled carbon nanotube thin films enriched with semiconducting nanotubes and their application in photoelectrochemical devices. Nanoscale 3, 1845-1849 (2011).

4. Liu, Z. \& Peng, R. Inorganic nanomaterials for tumor angiogenesis imaging. Eur J Nucl Med Mol Imaging 37 Suppl 1, S147-163 (2010).

5. Liang, F. \& Chen, B. A review on biomedical applications of single-walled carbon nanotubes. Curr Med Chem 17, 10-24 (2010).

6. Singh, R. \& Nalwa, H. S. Medical applications of nanoparticles in biological imaging, cell labeling, antimicrobial agents, and anticancer nanodrugs. J Biomed Nanotechnol 7, 489-503 (2011).

7. Rawson, F. J., Yeung, C. L., Jackson, S. K. \& Mendes, P. M. Tailoring 3D SingleWalled Carbon Nanotubes Anchored to Indium Tin Oxide for Natural Cellular Uptake and Intracellular Sensing. Nano Lett. (2012).

8. Bonner, J. C. Carbon nanotubes as delivery systems for respiratory disease: do the dangers outweigh the potential benefits? Expert Rev Respir Med 5, 779-787 (2011).

9. Wang, L. et al. Dispersion of single-walled carbon nanotubes by a natural lung surfactant for pulmonary in vitro and in vivo toxicity studies. Part Fibre Toxicol 7, 31 (2010).

10. Wadhwa, S. et al. Comparative in vitro cytotoxicity study of carbon nanotubes and titania nanostructures on human lung epithelial cells. J Hazard Mater 191, 56-61 (2011).

11. Zhang, Y. et al. Functionalized single-walled carbon nanotubes cause reversible acute lung injury and induce fibrosis in mice. J Mol Med (Berl) (2012).

12. Shvedova, A. A. et al. Impaired clearance and enhanced pulmonary inflammatory/ fibrotic response to carbon nanotubes in myeloperoxidase-deficient mice. PLoS One 7, e30923 (2012). 
13. Shvedova, A. A. et al. Unusual inflammatory and fibrogenic pulmonary responses to single-walled carbon nanotubes in mice. Am J Physiol Lung Cell Mol Physiol 289, L698-708 (2005).

14. Shvedova, A. A. \& Kagan, V. E. The role of nanotoxicology in realizing the helping without harm' paradigm of nanomedicine: lessons from studies of pulmonary effects of single-walled carbon nanotubes. J Intern Med 267, 106-118 (2010).

15. Zhao, X. \& Liu, R. Recent progress and perspectives on the toxicity of carbon nanotubes at organism, organ, cell, and biomacromolecule levels. Environ Int 40, 244-255 (2012).

16. Horie, M., Kato, H., Fujita, K., Endoh, S. \& Iwahashi, H. In vitro evaluation of cellular response induced by manufactured nanoparticles. Chem Res Toxicol 25, 605-619 (2012).

17. Samhan-Arias, A. K. et al. Oxidized phospholipids as biomarkers of tissue and cell damage with a focus on cardiolipin. Biochim Biophys Acta 1818, 2413-2423 (2012).

18. Gilmour, P. S. et al. Free radical activity of industrial fibers: role of iron in oxidative stress and activation of transcription factors. Environ Health Perspect 105 Suppl 5, 1313-1317 (1997).

19. Donaldson, K., Beswick, P. H. \& Gilmour, P. S. Free radical activity associated with the surface of particles: a unifying factor in determining biological activity? Toxicology Letters 88, 293-298 (1996).

20. Donaldson, K. et al. Free radical activity of PM10: iron-mediated generation of hydroxyl radicals. Environ Health Perspect 105 Suppl 5, 1285-1289 (1997).

21. Shvedova, A. A. et al. Exposure to carbon nanotube material: assessment of nanotube cytotoxicity using human keratinocyte cells. J Toxicol Environ Health A 66, 1909-1926 (2003).

22. Hurt, R. H., Monthioux, M. \& Kane, A. Toxicology of carbon nanomaterials: Status, trends, and perspectives on the special issue. Carbon 44, 1028-1033 (2006).

23. Makrygiannakis, D. et al. Citrullination is an inflammation-dependent process Ann Rheum Dis 65, 1219-1222 (2006).

24. Pawlik, A. et al. Allograft inflammatory factor-1 gene polymorphisms in patients with rheumatoid arthritis. Genet Test Mol Biomarkers 16, 341-345 (2012).

25. Chapuy-Regaud, S. et al. Fibrin deimination in synovial tissue is not specific for rheumatoid arthritis but commonly occurs during synovitides. J Immunol 174, 5057-5064 (2005).

26. Bongartz, T. et al. Citrullination in extra-articular manifestations of rheumatoid arthritis. Rheumatology 46, 70-75 (2007).

27. Mohamed, B. M. et al. Citrullination of proteins: a common post-translational modification pathway induced by different nanoparticles in vitro and in vivo. Nanomedicine (Lond) 7, 1181-1195 (2012).

28. Jones, C. F. \& Grainger, D. W. In vitro assessments of nanomaterial toxicity. $A d v$ Drug Deliv Rev 61, 438-456 (2009).

29. Plowden, J., Renshaw-Hoelscher, M., Engleman, C., Katz, J. \& Sambhara, S. Innate immunity in aging: impact on macrophage function. Aging Cell 3, 161-167 (2004).

30. Elkin, T. et al. Immuno-carbon nanotubes and recognition of pathogens. Chembiochem 6, 640-643 (2005).

31. Hitoshi, K., Katoh, M., Suzuki, T., Ando, Y. \& Nadai, M. Single-walled carbon nanotubes downregulate stress-responsive genes in human respiratory tract cells. Biol Pharm Bull 35, 455-463 (2012).
32. Baktur, R., Patel, H. \& Kwon, S. Effect of exposure conditions on SWCNT-induced inflammatory response in human alveolar epithelial cells. Toxicol In Vitro $\mathbf{2 5}$ 1153-1160 (2011).

33. $\mathrm{Hu}, \mathrm{X}$. et al. In vitro evaluation of cytotoxicity of engineered carbon nanotubes in selected human cell lines. Sci Total Environ 408 (2010).

34. Herzog, E. et al. SWCNT suppress inflammatory mediator responses in human lung epithelium in vitro. Toxicol Appl Pharmacol 234 (2009).

35. Knyazev, A. et al. Selective Adsorption of Proteins on Single-Wall Carbon Nanotubes by Using a Protective Surfactant. Chem. Eur. J. (2011).

36. Mohamed, B. M. et al. Activation of stress-related signalling pathway in human cells upon $\mathrm{SiO} 2$ nanoparticles exposure as an early indicator of cytotoxicity. J Nanobiotechnology 9, 29 (2011).

37. Movia, D. et al. Screening the Cytotoxicity of Single-Walled Carbon Nanotubes Using Novel 3D Tissue-Mimetic Models. ACS Nano 5, 9278-9290 (2011).

38. Byrne, F. et al. High content analysis of the biocompatibility of nickel nanowires. J Magn Magn Mater 321, 1341-1345 (2009).

39. Damoiseaux, R. et al. No time to lose--high throughput screening to assess nanomaterial safety. Nanoscale 3, 1345-1360 (2011).

40. Pruefer, F. G., Lizarraga, F., Maldonado, V. \& Melendez-Zajgla, J. Participation of Omi Htra2 serine-protease activity in the apoptosis induced by cisplatin on SW480 colon cancer cells. J Chemother 20, 348-354 (2008).

\section{Acknowledgments}

This work was supported by the EU FP7 NAMDIATREAM project (NMP-2009-LARGE-3-246479), and CRANN (CRANN Pathfinder to D.M.), A.K. for the financial support under EU FP7 NANOINTERACT project (NMP4-CT-2006-033231), HEA PRTLI cycles III-IV. B.M.M. for the financial support under MULTIFUN (NMP-2010-LARGE-4-246979) and INTERREG CAN project (INTERREG 4A IRELAND-WALES

\section{Author contributions}

B.M.M. and A.P.-M. conceptualized and planned the study; B.M.M., D.M. and A.P.-M carried out HCSA and ELISA experiments, analysed data and wrote the paper; A.K. and D.L. synthesized the chemically-treated SWCNTs; A.M.D. contributed to the optimization of the HCSA protocols; A.P.-M. and Y.V. coordinated the study. All authors discussed the results and commented on the paper.

\section{Additional information}

Supplementary information accompanies this paper at http://www.nature.com/ scientificreports

Competing financial interests: The authors declare no competing financial interests.

License: This work is licensed under a Creative Commons Attribution 3.0 Unported License. To view a copy of this license, visit http://creativecommons.org/licenses/by/3.0/

How to cite this article: Mohamed, B.M. et al. Citrullination as early-stage indicator of cell response to Single-Walled Carbon Nanotubes. Sci. Rep. 3, 1124; DOI:10.1038/srep01124 (2013) 